\title{
Wideband DoA Estimation Based on Joint Optimisation of Array and Spatial Sparsity
}

\author{
Mingyang Chen ${ }^{1}$, Wenwu Wang ${ }^{1}$, Mark Barnard ${ }^{1}$ and Jonathon Chambers ${ }^{2}$ \\ ${ }^{1}$ Department of Electrical and Electronic Engineering, University of Surrey, U.K. \\ Emails: \{m.chen; w.wang; mark.barnard $\} @$ surrey.ac.uk \\ ${ }^{2}$ School of Electrical and Electronic Engineering, Newcastle University, U.K. \\ Email: Jonathon.Chambers@newcastle.ac.uk
}

\begin{abstract}
We study the problem of wideband direction of arrival (DoA) estimation by joint optimisation of array and spatial sparsity. Two-step iterative process is proposed. In the first step, the wideband signal is reshaped and used as the input to derive the weight coefficients using a sparse array optimisation method. The weights are then used to scale the observed signal model for which a compressive sensing based spatial sparsity optimisation method is used for DoA estimation. Simulations are provided to demonstrate the performance of the proposed method for both stationary and moving sources.
\end{abstract}

\section{INTRODUCTION}

Source localisation, such as direction of arrival (DoA) estimation, is an important issue in a number of applications such as underwater acoustic detection, target tracking and environmental monitoring [1] [2]. The key to successful source localisation lies in the reliable extraction of useful information about the states of targets from the observations, which are collected by acoustic transducers, often operated in a noisy environment [2] [3]. Due to the constraints on computation resources, sensing range, communication bandwidth, and energy consumption, it is usually desirable not to use all the sensors in the array to report their measurements at all the time instants [4] [5] [6] [7]. This leads to the problem of sparse array optimisation and sensor selection, which seeks to activate a subset of sensors at different time to optimize the tracking performance under the given constraints.

Our work focuses on the problem of using as few sensors as possible to achieve DoA estimation at each sampling time. Traditionally, DoA estimation is addressed by methods, such as Capon beamformer, high-resolution and multiple signal classification (MUSIC) algorithm [8] [9] [10]. Recently, spatial sparsity based optimisation, which aims at extracting meaningful lower-dimensional information from high-dimensional data [11], has attracted great interests. Based on compressive sensing (CS) theory [12], the DoAs can be estimated by solving an optimisation problem constrained with the $l_{1}$ norm of a vector of the coefficients corresponding to the source activities in the spatial domain [13], which is assumed to be sparse, implying that only a few sources are active simultaneously. In these existing works, a full array has been used. However, as pointed out earlier, it would be desirable if the spatial sparsity can be used jointly with the array sparsity so that the sources can be localised with as few sensors from the array as possible.
This paper presents a joint array and spatial sparsity optimisation approach for wideband DoA estimation. The wideband signal is reconstructed as the combination of each sub-band to form a transverse line. The rebuilt signal can be regarded as a 'narrowband' input to a CS based two-step iterative process. With the joint approach, a fixed set of weight coefficients can be derived for each sensor, which are then used in the spatial sparsity based optimisation process for the estimation of DoAs. Different from the work in [14], whose wideband DoA estimation is achieved by exploiting joint sparsity of the divided spectral sub-bands of signals with a sensor array designed in advance, our approach exploits sparsity in both array and source space, and the sensor separation is calculated automatically based on given inputs. A two-layer Bayesian model was used in [15] based on sparse Bayesian learning [16], where a vector of hyper-parameters is used to control the common sparsity shared among different frequency bands in an underdetermined wideband scenario, and the expectation maximization algorithm is employed to estimate the DOA. However, in both layers, the problem of sparse signal recovery is considered, using a two-level nested array with fixed sensor elements. Therefore, this work does not consider the problem of sparse array optimisation and sensor selection. In [17], joint multicast beamforming and antenna selection is proposed to achieve the message propagation using the squared $\ell_{1}$-norm of the weight vector for the transmit-antenna elements, where the goal is to jointly select a limited number of antennas, and find their corresponding beamforming vectors so that the transmit power is minimized. However, the number of selected antennas is manually set in order to solve the induced non-convex optimisation problem, which is different from our method where this is found automatically as a result of the optimisation for the corresponding weights. In addition, the beamforming process based on the transmit power is different from the spatial sparsity based optimisation where a dictionary matrix is used to represent the received signal in a low-dimensional space.

This article is organised as follows. In Section II, the background including basic settings and related formulas are presented. The proposed method is presented in Section III. In Section IV, simulations on a stationary source, a moving source, noise added sources and a comparison with a baseline are presented. Finally, the conclusion is given in Section V. 


\section{BACKGROUND}

\section{A. Signal Model}

Suppose that at each time instant $k$, there is a wideband farfield source impinging on a linear array $\mathbf{x}_{k}=\left(x_{1}, \cdots, x_{M}\right)^{T}$ where $M$ is the number of possible source directions (i.e. DOA angles). The received sensor signal at each $k$ is denoted as $\mathbf{y}_{k}(j)=\left(y_{k 1}(j), \cdots, y_{k N}(j)\right)^{T}$, where $N$ is the number of sensors and $j=1, \ldots, J$ is the index of the frequency bands. We assume that the detection capability of each array element is unitary. In addition, source signals can only arrive in one half of the plane and the array is expected to have a perfect baffle, which means the arrival directions are from -90 degrees to +90 degrees along the plane of the array elements, and 0 degree is the normal to the line of the array [18].

A dictionary matrix $\mathbf{A}$ is created by sampling all possible DoAs for the wideband array. The $n m$-th element of $\mathbf{A}$ for the $j$-th frequency band is defined as

$$
\mathbf{A}_{n m}(j)=\exp \left[i 2 \pi f(j) d_{n} c^{-1} \sin \theta_{m}\right]
$$

where $i \equiv \sqrt{-1}, d_{n}$ denotes the distance between the $n$ th sensor and the first sensor for $n=1,2, \cdots, N, c$ is the speed of wave propagation, and $\theta_{m}$ is the DoA of the $m$-th hypothetical source to the array for the $j$-th frequency band $f(j)$, where $m=1,2, \cdots, M$.

For each vector $\mathbf{x}_{k}$, the $N$ dimensional array output of the $j$-th frequency band is expressed as

$$
\mathbf{y}_{k}(j)=\mathbf{A}(j) \mathbf{x}_{k}+\mathbf{n}_{k}(j)
$$

where $\mathbf{n}_{k}(j)$ is a random noise vector at the $j$-th frequency band. Here we consider isotropic noise in the assumed half plane as used in our experiments.

\section{B. CS-Based Sparse Array}

In a sparse array, the aim is to use as few sensors as possible to achieve similar performance to that via the full array. For the narrowband case, this can be formed as a CS problem by minimising the $\ell_{1}$ norm of the weight coefficients [19], as follows,

$$
\begin{gathered}
\min \|\mathbf{w}\|_{1} \\
\text { subject to }\left\|\mathbf{p}^{T}-\mathbf{w}^{H} \mathbf{A}\right\|_{2} \leq \alpha
\end{gathered}
$$

where $\mathbf{w} \in \mathbb{C}^{N}$ is the coefficient vector of the array, $\mathbf{p} \in \mathbb{C}^{M}$ is the vector holding the desired beam response at the sampled angular points $\theta_{m}$ for the frequency of interest $\Omega, \alpha \in \Re^{+}$is a threshold measuring the similarity between the designed response and the desired response, $(\cdot)^{H}$ is a Hermitian operator, $\|\cdot\|_{1}$ and $\|\cdot\|_{2}$ are respectively the $\ell_{1}$ and $\ell_{2}$ norm of their arguments. Here, $\mathbf{p}$ is defined by

$$
\mathbf{p}=\left[p\left(\Omega, \theta_{1}\right), p\left(\Omega, \theta_{2}\right), \cdots, p\left(\Omega, \theta_{M}\right)\right]^{T}
$$

where $p(\Omega, \theta)$ is a desired response at the direction $\theta$ and frequency $\Omega$, which, in the wideband case, can be replaced as $\Omega_{j}$ for the $j$-th frequency band.

\section{Spatial Sparsity based DoA Estimation}

Different from the sparse array problem, in spatial sparsity based DoA estimation, the aim is to find the weight that corresponds to the source direction based on a full array, assuming that the number of active sources is small as compared to the total number of possible source directions. This can be achieved by a sequential Bayesian technique based on the least absolute shrinkage and selection operator (LASSO), as proposed in [20], [21]. For the narrowband signal $\mathbf{y}_{k}$ at time $k$ and source activation vector $\mathbf{x}_{k}$, the cost function to be minimised is given as follows,

$$
\underset{\mathbf{x}_{k}}{\operatorname{argmin}}\left\|\mathbf{y}_{k}-\mathbf{A} \mathbf{x}_{k}\right\|_{2}^{2}+\mu\left\|\mathbf{D} \mathbf{x}_{k}\right\|_{1}
$$

with

$$
\begin{gathered}
\mathbf{D}=\sigma^{2} \mathbf{V} \\
\mathbf{V}=\operatorname{diag}(\mathbf{v})
\end{gathered}
$$

where $\mathbf{D}$ and $\mathbf{V}$ are the matrices holding the coefficients vector $\mathbf{v}=\left(v_{1}, v_{2}, \ldots, v_{\mathrm{M}}\right)^{T}$, which corresponds to the source activity in the source space, $\mu$ is a regularization parameter, and $\sigma^{2}$ is the noise variance. For the broadband scenario, the relative levels of added noise are different at each frequency band. As a result, the value of $\sigma$ can be taken as the average of relative levels.

The weights can be estimated sequentially, i.e. the result at the $(k+1)$-th time step $v_{m}[k+1]$ should be calculated using $v_{m}[k]$ at the $k$-th time step. In the neighbourhood of an active source defined by a threshold [21] [22], the predicted weights $v_{m}[k+1]$ are calculated as below:

$$
\left(v_{m}[k+1]\right)^{2}=\left(\sum_{j=-l}^{l} \frac{\alpha_{j}}{\left(v_{m+j}[k]\right)^{2}}\right)^{-1}
$$

with non-negative coefficients $\alpha_{j}, \sum_{j} \alpha_{j}=1$ and the weights in $\mathbf{v}$ are bounded by 1 . In practice, a small threshold e.g. $100 \times$ $2^{-52}$ is used to prevent the value of $v_{m+j}[k]$ from approaching zero in order to avoid invalid division. When the estimation is out of the neighbourhood of the active source, the weight prediction is replaced by:

$$
v_{m}[k+1]=v_{m}[k]+c v_{0}
$$

where $v_{0}$ and $c$ are defined empirically [21] [22].

\section{JoInt Sparsity BASED Method For WidebAND DOA ESTIMATION}

In this section, we present a method for wideband DoA estimation by exploiting the spatial sparsity and using as few sensors from the array as possible. This method is actually an extension of our recent work in [18], which is a two-step process designed for narrow-band DoA estimation. With the input of a given beam, the first step is to implement the CSbased sparse array algorithm through the cost function (3) to obtain a set of sparse sensor weight coefficients. The second step is to plug the estimated weight coefficients into the cost 
function (5), as a scaling factor for $\mathbf{y}_{k}$. The cost function for the joint sparsity approach is defined as

$$
\underset{\mathbf{x}_{k}, v_{m}}{\operatorname{argmin}}\left\|\mathbf{W} \mathbf{y}_{k}-\mathbf{A} \mathbf{x}_{k}\right\|_{2}^{2}+\mu\left\|\mathbf{D} \mathbf{x}_{k}\right\|_{1}
$$

where $\mathbf{W}$ is defined as

$$
\mathbf{W}=\left(\begin{array}{lll}
\left|w_{1}\right| & & \\
& \ldots & \\
& & \left|w_{N}\right|
\end{array}\right)
$$

For the wideband scenario, the signal needs to be reshaped as a narrowband-like input. With this input the sparse array optimisation is used to calculate the weight coefficients for each sensor for different frequency bands. Then the weight coefficients are restructured to match the length of $\mathbf{y}_{k}$ and to scale the expanded $\mathbf{y}_{k}$. The spatial sparsity based technique is then used to derive the DoA before reshaping it back to the wideband set up.

\section{A. Reshaping Step}

For the sparse array optimisation, in order to implement the cost function (3) for wideband signals, the response matrix $\mathbf{P} \in \mathbb{C}^{M \times J}$ can be reshaped to an $M J$ dimensional vector, $\mathbf{p}_{\text {reshape }} \in \mathbb{C}^{M J}$, as follows,

$$
\begin{aligned}
\mathbf{p}_{\text {reshape }}= & {\left[p\left(\Omega_{1}, \theta_{1}\right), \cdots, p\left(\Omega_{1}, \theta_{M}\right), \cdots,\right.} \\
& p\left(\Omega_{2}, \theta_{1}\right), \cdots, p\left(\Omega_{2}, \theta_{M}\right), \cdots, \\
& \left.p\left(\Omega_{J}, \theta_{1}\right), \cdots, p\left(\Omega_{J}, \theta_{M}\right)\right]^{T}
\end{aligned}
$$

Corresponding to the desired beam response, the dictionary matrix $\mathbf{A}$ whose elements are defined in (1) for sparse array optimisation is changed to $\mathbf{A}_{\text {array }} \in \mathbb{C}^{N \times(M J)}$, as follows

$$
\begin{aligned}
\mathbf{A}_{\text {array }}=\exp [ & i 2 \pi f(1) d_{n} c^{-1} \sin \theta_{m}, \\
& i 2 \pi f(2) d_{n} c^{-1} \sin \theta_{m}, \cdots, \\
& \left.i 2 \pi f(J) d_{n} c^{-1} \sin \theta_{m}\right]
\end{aligned}
$$

Similar to sparse array optimisation, the observed signal $\mathbf{y}_{k}$ used in spatial sparsity based DoA estimation is reshaped as

$$
\mathbf{y}_{k-\text { reshape }}=\left[\mathbf{y}_{k}(1), \mathbf{y}_{k}(2), \cdots, \mathbf{y}_{k}(J)\right]^{T}
$$

The dictionary matrix $\mathbf{A}$ used in the spatial sparsity based DoA estimation becomes $\mathbf{A}_{\text {spatial }} \in \mathbb{C}^{(N J) \times M}$ defined as

$$
\begin{aligned}
\mathbf{A}_{\text {spatial }} & =\exp \left[\left(i 2 \pi f(1) d_{n} c^{-1} \sin \theta_{1}, \cdots,\right.\right. \\
& \left.i 2 \pi f(J) d_{n} c^{-1} \sin \theta_{1}\right)^{T},\left(i 2 \pi f(1) d_{n} c^{-1} \sin \theta_{2}, \cdots,\right. \\
& \left.i 2 \pi f(J) d_{n} c^{-1} \sin \theta_{2}\right)^{T},, \cdots,\left(i 2 \pi f(1) d_{n} c^{-1} \sin \theta_{M}, \cdots\right. \\
& \left.\left.i 2 \pi f(J) d_{n} c^{-1} \sin \theta_{M}\right)^{T}\right]
\end{aligned}
$$

\section{B. Sparse Array Optimisation Step}

For the CS-based sparse wideband array optimisation, the cost function (3) with complex vector weight coefficients, i.e. $\mathbf{w}=\mathbf{w}_{R}+\mathbf{w}_{I} i$, is modified as

$$
\begin{gathered}
\min \left\|\mathbf{w}_{R}\right\|_{1}+\left\|\mathbf{w}_{I}\right\|_{1} \\
\text { subject to }\left\|\mathbf{p}_{\text {reshape }}^{T}-\left(\mathbf{w}_{R}+\mathbf{w}_{I} i\right)^{H} \mathbf{A}_{\text {array }}\right\|_{2} \leq \alpha
\end{gathered}
$$

where $\mathbf{p}_{\text {reshape }}$ is the reshaped input vector holding the desired beam response at source directions and $\mathbf{A}_{\text {array }}$ is the reshaped dictionary matrix matching the size of the re-shaped response vector.

The length of the obtained $\mathbf{w}$ is $N$. Before performing the spatial sparsity based DoA estimation, the weight coefficients for all the frequency bands are structured to match the size of $\mathbf{y}_{k-\text { reshape }}$ as follows

$$
\mathbf{w}_{\text {repeat }}=\left[\mathbf{w}_{1}, \mathbf{w}_{2}, \cdots, \mathbf{w}_{J}\right]^{T}
$$

\section{Spatial Sparsity based DoA Estimation Step}

After obtaining the suitable $\mathbf{w}_{\text {repeat }}$, the LASSO function (5) is then modified as

$\underset{\mathbf{x}_{k}, \operatorname{argmin}}{\operatorname{ar}}\left\|\mathbf{W}_{\text {reshape }} \mathbf{y}_{k-\text { reshape }}-\mathbf{A}_{\text {spatial }} \mathbf{x}_{k}\right\|_{2}^{2}+\mu\left\|\mathbf{D} \mathbf{x}_{k}\right\|_{1}$ $\mathbf{x}_{k}, v_{m}$

where $\mathbf{W}_{\text {reshape }} \in \mathbb{C}^{(N J) \times(N J)}$ is defined as

$$
\mathbf{W}_{\text {reshape }}=\operatorname{diag}\left(\left|\mathbf{w}_{\text {repeat }}\right|\right)
$$

The obtained $\mathbf{x}_{k} \in \mathbb{C}^{M}$ is then used to construct $\mathbf{x}_{k \text {-repeat }} \in \mathbb{C}^{(M J)}$, as below

$$
\mathbf{x}_{k-\text { repeat }}=\left[\mathbf{x}_{k}, \cdots, \mathbf{x}_{k}\right]^{T}
$$

For the iterative implementation, the estimated $\mathbf{x}_{k}$ is then used to reconstruct the response vector (21) and $\mathbf{D}$ is updated as (6). The above two processes are iterated until the DoA is found using a minimum number of sensors.

$$
\mathbf{p}=\left[\left(\mathbf{A}_{\text {array }} \mathbf{x}_{k-\text { repeat }}\right)^{H} \mathbf{A}_{\text {array }}\right]^{T}
$$

Both cost functions (16) and (18) are optimised by the CVX toolbox in Matlab [23].

The procedure of the proposed joint sparse array and spatial sparsity based optimisation for wideband DoA estimation is shown in Table I, where the number of iterations in the inner loop $k k$ could be increased to achieve better performance. The input beam response $\mathbf{p}$ can be initialised randomly, e.g. at 0 degree.

\section{Simulation Experiments And Discussions}

In this section, we illustrate the performance of the proposed joint sparsity based method for wideband DoA estimation. All the experiments are based on simulated data, which consists of a stationary source at 20 degrees and a moving source whose DoAs changes gradually from 43 degrees to -9 degrees. For each experiment, the first input beam response is initialised at 0 degree based on a Chebyshev window function (simply set to be different from the ground truth of the source direction to show the capability of the proposed method). The incidence source angle is assumed to be the same for each frequency band and the value is changed at a constant rate, therefore no Doppler shift is considered in this paper.

The speed of underwater sound used in this approach is assumed to be $1500 \mathrm{~m} / \mathrm{s}$, and the wideband frequency of the sources is divided into bands at $1000 \mathrm{~Hz}, 1100 \mathrm{~Hz}, 1200 \mathrm{~Hz}$, $1300 \mathrm{~Hz}$, and $1400 \mathrm{~Hz}$. A linear array with 300 sensors is used 


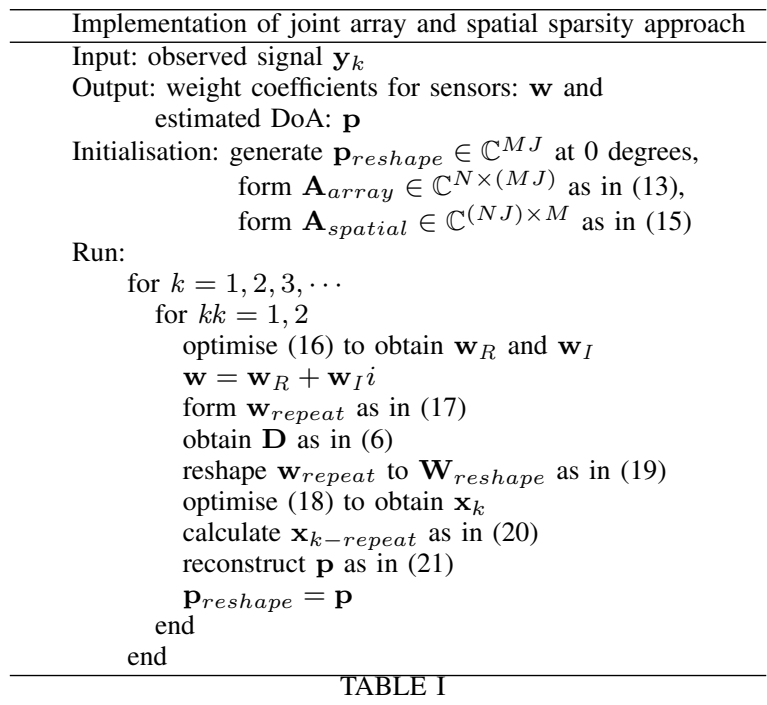

THE PROPOSED JOINT SPARSITY BASED OPTIMISATION METHOD

\begin{tabular}{|c|c|c|}
\hline & Stationary source & Moving source \\
\hline$M S E_{\text {array }}(\mathrm{dB})$ & -52.6 & -53.7 \\
\hline$M S E_{\text {spatial }}(\mathrm{dB})$ & -39.8 & -39.1 \\
\hline No. of active sensors & 64 & 69 \\
\hline
\end{tabular}

SIMULATION RESULTS FOR STATIONARY SOURCE AND MOVING SOURCE.

and the inter-sensor spacing is $0.05 \lambda$ ( $\lambda$ is the wavelength). Here the value of sensor separation is chosen empirically according to the scale of the array. The maximum running time-step is $K=20$. The number of steps is set to ensure convergence. The constraint value of $\alpha$ used in (16) is 0.3 , which controls how close the designed response is to the desired response. The Mean Square Errors (MSEs) for sparse array optimisation and spatial sparsity based optimisation are used as the performance index, which are defined according to functions (16) and (21) as

$$
\begin{gathered}
M S E_{\text {array }}=20 \log _{10}\left(\frac{\left\|\mathbf{p}_{\text {reshape }}^{T}-\mathbf{w}^{H} \mathbf{A}_{\text {array }}\right\|_{2}^{2}}{M}\right) d B \\
M S E_{\text {spatial }}=20 \log _{10}\left(\frac{\left\|\mathbf{y}_{k}^{H} \mathbf{A}-\mathbf{p}^{T}\right\|_{2}^{2}}{M}\right) d B
\end{gathered}
$$

where $M$ is the number of potential source directions. The results of $\mathbf{y}_{k}^{H} \mathbf{A}$ obtained through calculating each frequency band are combined to match the size of $\mathbf{p}$. The average values for both MSEs are calculated with the total running step of $K$.

For the sources without noise, the simulation results shown in Table II illustrate a close match between the desired signal $\mathbf{p}_{\text {reshape }}$ and the designed signal $\mathbf{w}^{H} \mathbf{A}_{\text {array }}$ as defined by (22). The values of MSEs for the stationary source are similar to those for the moving source, but for the moving source, more sensors need to be used under the same level of noise.

To demonstrate the influence of noise, noise at the levels 25 $\mathrm{dB}, 30 \mathrm{~dB}$ and $40 \mathrm{~dB}$ in terms of Signal to Noise Ratio (SNR) is added to the sensor signals. The noise is isotropic around

\begin{tabular}{|c|c|c|c|c|}
\hline & $\begin{array}{c}M S E_{\text {array }} \\
(\mathrm{dB})\end{array}$ & $\begin{array}{c}M S E_{\text {spatial }} \\
(\mathrm{dB})\end{array}$ & $\begin{array}{c}\text { Active } \\
\text { sensors }\end{array}$ & $\begin{array}{c}\text { SNR } \\
(\mathrm{dB})\end{array}$ \\
\hline Stationary source & -45.9 & -34.6 & 61 & 30 \\
\hline Moving source & -45.4 & -38.8 & 64 & 30 \\
\hline Moving source & -31.5 & -26.8 & 75 & 25 \\
\hline Moving source & -57.0 & -39.9 & 60 & 40 \\
\hline \multicolumn{4}{|r}{ TABLE III }
\end{tabular}

SIMULATION RESULTS FOR NOISE ADDED SOURCES.

the half space and follows the i.i.d. Gaussian distribution with zero mean and unit variance. The results are shown in Table III. From this table, we can see that when the noise level is identical, the moving source tends to use more sensors as compared with the stationary source. In both cases, the MSEs are larger than those without noise. As the SNR increases, the MSEs decrease and fewer active sensors are used. The experiments for both the stationary source and moving source demonstrate a satisfactory performance of the joint approach.

We also compare the joint approach with a baseline which only considers the spatial sparsity-based optimisation using a full array. The $M S E_{\text {spatial }}$ results at each time-step are shown in Figure 1. In this experiment, we only process noiseless signals at frequency bands of $1000 \mathrm{~Hz}$ and $1100 \mathrm{~Hz}$ and the total running time $K=100$. It can be seen that the joint optimisation method gives similar DoA estimation results to those in the spatial sparsity based baseline. The minimum value is almost the same as $-164 \mathrm{~dB}$ for both methods at the time step of $k=84$. From Figure 2 we can observe that after $k=84$, there is less estimation noise than the interval from $k=40$ to $k=80$. The comparison experiment shows that the joint approach can achieve DoA estimation similar to the single spatial sparsity optimisation but with much fewer sensors.

\section{CONClusion}

We have presented a new algorithm for wideband DoA estimation based on joint optimisation of array and spatial sparsity. The method operates by reshaping the wideband signal which is then used in CS-based sparse array optimisation to estimate the weight coefficients for the selection of the sensors. The obtained weight coefficients are then used to derive the DoA of the sources based on spatial sparsity. The procedure is iterated in an alternating manner. The algorithm starts from an initialized input and the approach can adjust itself to obtain the DoA estimation. The results evaluated for both a stationary source and a moving source show good performance of the proposed method. For the future work, we will consider the scenarios of multiple sources with larger bandwidth and stronger noise, typically happening in underwater applications. A comparison to other conventional DOA estimation methods will also be considered. 


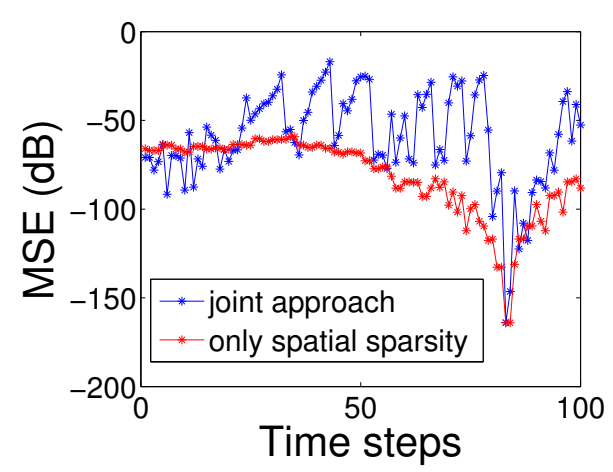

Fig. 1. MSE $E_{\text {spatial }}$ for the moving source without noise.

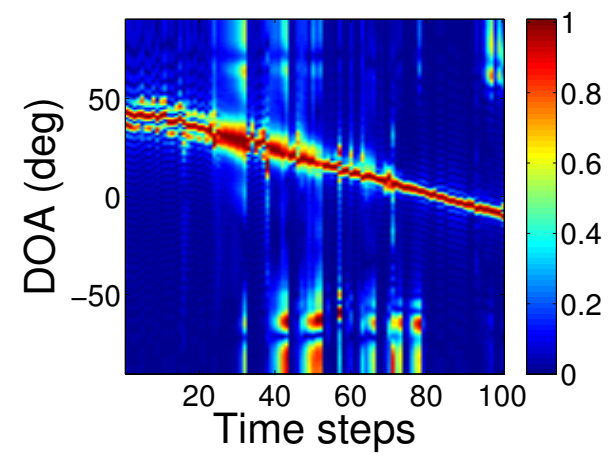

Fig. 2. DoAs (p) estimated for the moving source without noise at the frequency band of $1100 \mathrm{~Hz}$.

\section{REFERENCES}

[1] J. Benesty, J. Chen, and Y. Huang, Microphone array signal processing. Springer Science \& Business Media, 2008, vol. 1.

[2] M. R. Bai, J.-G. Ih, and J. Benesty, Acoustic Array Systems: Theory, Implementation, and Application. John Wiley \& Sons, 2013.

[3] M. E. G. Borges, D. Maltese, P. Vanheeghe, G. Sella, and E. Duflos, "Sensor management using expected risk reduction approach," in 2016 19th International Conference on Information Fusion (FUSION). IEEE, 2016, pp. 2050-2058.

[4] S. Liu, F. Chen, A. Vempaty, M. Fardad, L. Shen, and P. K. Varshney, "Sparsity-promoting sensor management for estimation: An energy balance point of view," in 2015 18th International Conference on Information Fusion (Fusion). IEEE, 2015, pp. 231-238.

[5] L. Zuo, R. Niu, and P. Varshney, "Posterior crlb based sensor selection for target tracking in sensor networks," in ICASSP 2007. IEEE International Conference on Acoustics, Speech and Signal Processing, vol. 2. IEEE, 2007, pp. II-1041.

[6] R. Tharmarasa, T. Kirubarajan, and M. Hernandez, "Large-scale optimal sensor array management for multitarget tracking," IEEE Transactions on Systems, Man, and Cybernetics, Part C (Applications and Reviews), vol. 37, no. 5, pp. 803-814, 2007.

[7] R. Tharmarasa, T. Kirubarajan, A. Sinha, and T. Lang, "Decentralized sensor selection for large-scale multisensor-multitarget tracking," IEEE Transactions on Aerospace and Electronic systems, vol. 47, no. 2, pp. 1307-1324, 2011.

[8] P. Händel, P. Stoica, and T. Söderström, "Capon method for doa estimation: accuracy and robustness aspects," in Proceedings of The IEEE Winter Workshop on Nonlinear Digital Signal Processing. IEEE, 1993, pp. P-7.

[9] P. Totarong and A. El-Jaroudi, "A novel approach for robust highresolution doa estimation," in Proceedings of The IEEE Sixth SP Workshop on Statistical Signal and Array Processing. IEEE, 1992, pp. 366-369.

[10] A. Hakam, R. M. Shubair, and E. Salahat, "Enhanced doa estimation algorithms using mvdr and music," in 2013 International Conference on Current Trends in Information Technology (CTIT). IEEE, 2013, pp. $172-176$.

[11] P. Knee, "Sparse representations for radar with MATLAB $\AA$ examples," Synthesis Lectures on Algorithms and Software in Engineering, vol. 4, no. 1, pp. 1-85, 2012.

[12] D. L. Donoho, "Compressed sensing," IEEE Transactions on Information Theory, vol. 52, no. 4, pp. 1289-1306, 2006.

[13] D. Malioutov, M. Çetin, and A. S. Willsky, "A sparse signal reconstruction perspective for source localization with sensor arrays," IEEE Transactions on Signal Processing, vol. 53, no. 8, pp. 3010-3022, 2005.

[14] L. Wang, L. Zhao, G. Bi, and C. Wan, "Wideband DOA estimation by joint sparse representation under bayesian learning framework," in 2015 IEEE International Conference on Digital Signal Processing (DSP). IEEE, 2015, pp. 366-369.

[15] N. Hu, B. Sun, Y. Zhang, J. Dai, J. Wang, and C. Chang, "Underdetermined doa estimation method for wideband signals using joint nonnegative sparse bayesian learning," IEEE Signal Processing Letters, vol. 24, no. 5, pp. 535-539, 2017.

[16] N. Hu, B. Sun, J. Wang, J. Dai, and C. Chang, "Source localization for sparse array using nonnegative sparse bayesian learning," Signal Processing, vol. 127, pp. 37-43, 2016.

[17] O. Mehanna, N. D. Sidiropoulos, and G. B. Giannakis, "Multicast beamforming with antenna selection," in 2012 IEEE 13th International Workshop on Signal Processing Advances in Wireless Communications (SPAWC). IEEE, 2012, pp. 70-74.

[18] M. Chen, M. Barnard, and W. Wang, "Joint array and spatial sparsity based optimisation for DoA estimation," in IEEE Sensor Signal Processing for Defence Conference. IEEE, 2016.

[19] M. B. Hawes and W. Liu, "Robust sparse antenna array design via compressive sensing," in 2013 18th International Conference on Digital Signal Processing. IEEE, 2013, pp. 1-5.

[20] C. F. Mecklenbrauker, P. Gerstoft, A. Panahi, and M. Viberg, "Sequential bayesian sparse signal reconstruction using array data," IEEE Transactions on Signal Processing, vol. 61, no. 24, pp. 6344-6354, 2013.

[21] E. Zochmann, P. Gerstoft, and C. F. Mecklenbrauker, "Density evolution of sparse source signals," in 2015 3rd International Workshop on Compressed Sensing Theory and its Applications to Radar, Sonar and Remote Sensing. IEEE, 2015, pp. 124-128.

[22] M. Barnard and W. Wang, "Adaptive Bayesian sparse representation for underwater acoustic signal denoising," in Proc. 2nd IET International Conference on Intelligent Signal Processing, 2015.

[23] M. Grant, S. Boyd, and Y. Ye, "CVX: MATLAB software for disciplined convex programming," 2008. 